\title{
Touristen auf der mexikanischen Halbinsel Yucatán im Kreuzfeuer der Übersetzung: eine Übersetzungskritik von Javier Covo Torres' Los mayas en las rocas
}

[Mexico's Yucatan Peninsula Tourists in the Crossfire of Translation: a Translation Criticism of Los mayas en las rocas by Javier Covo Torres]

http://dx.doi.org/10.11606/1982-8837213524

\author{
Dorit Heike Gruhn ${ }^{1}$ \\ Gaspar Ramírez Cabrera ${ }^{2}$ \\ Karin Kottenhahn ${ }^{3}$
}

\begin{abstract}
Since the advent of the functional approach in translation science, any translation criticism has to take into account the target group of a translation. This is to be our starting point to analyze the Spanish-German and Spanish-English translations of Los mayas en las rocas by Javier Covo Torres, an illustrated book that seeks to give a didactic introduction to the culture of the pre-Hispanic Maya and is aimed mainly at tourists who visit the Yucatan Peninsula. The main objective of this work is to evaluate the adequacy of translations in relation to their addresses. In order to accomplish this objective, we rely methodologically on some of the parameters of Silvia Parra Galiana, who proposes a review of a translation in two phases, covering different criteria. We devote special attention to the interrelation between text and illustrations (including mini-cartoons), since the latter represent a special challenge for the translator. The findings will be placed within a context of editorial decisions taken far from the knowledge and discussions of academic discipline.
\end{abstract}

Keywords: translation criticism, target-group-specific, translation and illustration, Maya

Zusammenfassung: Seit dem funktionalen Ansatz in der Übersetzungswissenschaft ist die Frage nach der Zielgruppe aus der Übersetzungskritik nicht mehr wegzudenken. Das ist unser Ausgangspunkt bei der Untersuchung der Spanisch-Deutsch und Spanisch-Englisch-Übersetzung von Los mayas en las

\footnotetext{
${ }^{1}$ Benemérita Universidad Autónoma de Puebla - Facultad de Lenguas, 24 Norte 2003 Colonia Humboldt, Puebla, Puebla 72370, México. E-mail: heike50@hotmail.com.

${ }^{2}$ Benemérita Universidad Autónoma de Puebla - Facultad de Lenguas, 24 Norte 2003 Colonia Humboldt, Puebla, Puebla 72370, México. E-mail: gasparfirst@gmail.com.

${ }^{3}$ Institut für Fremdsprachen und Auslandskunde bei der Universität Erlangen-Nürnberg - Spanischabteilung, Hindenburgstraße 42, Erlangen, Bayern 91054, Germany. E-mail: karin.kottenhahn@ fen-net.de.
}

\section{(cC) BY-NC}


Gruhn, D.H.; Cabrera, G.R.; Kottenhahn, K. - Touristen auf Yucatán

rocas des Autors Javier Covo Torres. Dabei handelt es sich um ein für Touristen konzipiertes Buch, das eine didaktisierte Einführung in die Kultur der prähispanischen Maya geben möchte. Übergeordnetes Untersuchungsziel ist die Bewertung der Adäquatheit der Zieltexte, wobei wir uns methodisch auf einige Parameter der Übersetzungsbewertung von Silvia Parra Galiana stützen, die eine Überprüfung in zwei Phasen vorschlägt, welche unterschiedliche Kriterien abdecken. Unser besonderes Augenmerk gilt dabei dem Zusammenspiel von Text und Illustrationen, denn letztere stellen den Übersetzer vor besondere Herausforderungen. Die Ergebnisse stellen wir in Zusammenhang mit verlegerischen Entscheidungen, die fernab des Wissens- und Diskussionsstands der akademischen Disziplin getroffen werden.

Stichwörter: Übersetzungskritik, Zielgruppenspezifik, Übersetzung und Illustrationen, Maya

\section{Einführung}

Im Jahr 2016 reisten rund 35 Millionen Ausländer nach Mexiko ein. Eine der touristischen Hauptattraktionen des Landes ist die Halbinsel Yucatán, auf der es - neben weiteren Sehenswürdigkeiten - unzählige archäologische Maya-Stätten zu besichtigen gibt; es ist davon auszugehen, dass die dortige Besucherzahl ebenfalls im zweistelligen Millionenbereich liegt ${ }^{4}$. Mancher Reisende ist daran interessiert, sich näher mit Geschichte und Kultur der Gegend auseinanderzusetzen. In den Buchhandlungen lassen sich folglich diverse Veröffentlichungen auf Englisch, Deutsch, Französisch und in anderen Sprachen finden - die meisten sind Übersetzungen von Texten, deren Originalversion für mexikanische Touristen und Kulturinteressierte geschrieben wurde.

Doch bringen diese Übersetzungen ihren Lesern die fremde Welt wirklich näher? Oder anders gefragt: Welchen Ansprüchen sollten solche Übersetzungen genügen? Am Beispiel einer vergleichenden Übersetzungskritik Spanisch-Deutsch und Spanisch-Englisch des illustrierten Büchleins Los mayas en las rocas von Javier Covo Torres möchte der vorliegende Artikel einige Kriterien zur Bewertung von Übersetzungen im touristischen Bereich nachzeichnen. Die übergeordnete Fragestellung dieser Untersuchung beschäftigt sich mit der Adäquatheit der Zieltexte (nachfolgend: ZT), das heißt mit der Frage, wie angemessen die zwei Übersetzungen in Hinblick auf ihre Adressaten sind. Der Schwerpunkt unserer Analyse liegt dabei auf der deutschsprachigen Version mit dem Titel Die Mayas in der Steinzeit (Covo TORRES 2007, Ü. v. Kerstin Regel de Baena), die englischsprachige The mayas on the rocks (COvo TORRES 2010, Ü. v. David Phillips) wird als Kontrastversion mit hinzugezogen. Darüber

\footnotetext{
4 <http://www.datatur.sectur.gob.mx/SitePages/VisitantesInternacionales.aspx>. (20.08.2017). Für die Halbinsel Yucatán stehen keine entsprechenden Daten zur Verfügung.
} 
Gruhn, D.H.; Cabrera, G.R.; Kottenhahn, K. - Touristen auf Yucatán

hinaus verfolgt dieser Artikel auch didaktische Zwecke: Angehende Übersetzer können daran nachvollziehen, welche Fragestellungen die Übersetzung eines solchen - nur auf den ersten Blick einfachen - Textes aufwerfen kann und welche Fehlerquellen sie verbirgt.

\section{Der Ausgangstext und seine Adressaten}

In Anlehnung an Christiane Nord (1991: 32) gehen wir von einer bilateralen Gebundenheit des Übersetzers aus: "Der Translator ist demnach bilateral gebunden: an den Ausgangstext und an die Ziel(text)situation, und er trägt Verantwortung sowohl gegenüber dem AT-Sender [...] als auch gegenüber dem Zieltextempfänger" (eine Verantwortung, die Nord bekannterweise als Loyalität bezeichnet). Das verpflichtet uns, zunächst einmal den Ausgangstext (nachfolgend: AT) und dessen Adressaten grob zu umreißen.

Der Verlag Editorial Dante nennt sich selbst "La Editorial del Mundo Maya" und widmet sich, eigenen Angaben zufolge, unter anderem touristischen und didaktischen Veröffentlichungen, die in der hauseigenen Buchhandlungskette in mehreren Zweigstellen auf Yucatán vertrieben werden, ebenso an einigen der großen archäologischen Stätten. Hier können also sowohl der spanischsprachige Titel als auch die Übersetzungen erworben werden ${ }^{5}$; darüber hinaus sind sie als Kindle-E-Book im Internet erhältlich.

In 28 sehr kurzen Kapiteln gibt Los mayas en las rocas eine didaktisierte Einführung in verschiedene Themen der prähispanischen Maya (Religion, Feste, Mathematik, Handel u.a.).

Wer sind nun die Adressaten des Büchleins? "Als Adressaten sind jene Personen zu verstehen, an die der Autor dachte, als er den Text abfasste. Diese Adressaten sind also nicht zwangsweise deckungsgleich mit den tatsächlichen Lesern oder Rezipienten, das heißt mit denjenigen Personen, die ein bestimmtes Dokument irgendwann wirklich lesen" (HENNEQUIN 1999: 41, Ü. H.G.). Im vorliegenden Text verwenden wir auch die Begriffe (potentielle) Rezipienten oder Zielgruppe als Synonyme zu Adressaten.

Den Verkaufsstellen nach zu urteilen, richtet sich das Buch vorrangig an mexikanische Touristen der Halbinsel Yucatán. Inhalt und Sprache verstärken diesen Eindruck, denn Konzepte, die dem mexikanischen Leser allgemein vertraut sind (juego de pelota, mexicas u.a.) werden nicht erläutert, und die Sprachform (vor allem der mündlichen Rede in den Cartoons)

\footnotetext{
${ }^{5}$ Die Übersetzung ins Deutsche ist allerdings nur noch schwer erhältlich.

Pandaemonium, São Paulo, v. 21, n. 35, set.-dez. 2018, p. 24-46
} 
Gruhn, D.H.; Cabrera, G.R.; Kottenhahn, K. - Touristen auf Yucatán

ist an das mexikanische Spanisch angepasst (Personalpronomen "tú", Verbformen), wenngleich auch für Sprecher anderer hispanoamerikanischer Länder verständlich.

Die Rezipienten werden wahrscheinlich auf Yucatán unterwegs sein, wenn sie das Büchlein durchblättern (z.B. in einem Hotelzimmer oder am Strand), weshalb die Lektüre nicht allzu anspruchsvoll oder anstrengend sein darf. Der Text ist diesem Umstand durchaus angepasst: Register und Stil können folgendermaßen beschrieben werden: Standardsprache mit sehr einfacher Syntax, die einzelnen Absätze bestehen aus nicht mehr als zwei oder drei Sätzen, wobei diese Sätze meist sehr kurz sind. Der Text enthält eine Vielzahl an Begriffen auf Maya (Götter, Amtsträger, Kalendertage u.a.), für die i.d.R. keine Übersetzung ins Spanische existiert, sowie einige architektonische Fachbegriffe. Die unbekannten Konzepte werden fast immer im Text erklärt bzw. mit Zeichnungen veranschaulicht. Ein Maya-Spezialist im Forschergewand führt durch das gesamte Buch, dieser sieht im Übrigen dem in Mérida ansässigen Autor kolumbianischer Herkunft ähnlich. Seine Kommentare erscheinen meist in Sprechblasen ${ }^{6}$.

Das Buch wendet sich somit an ein breites Publikum und ist für Leser ab ungefähr 12 Jahren verständlich. Dem Umstand, dass die prähispanischen Maya in Mexiko auch Stoff des Schulunterrichts sind, was vor allem bei jüngeren Lesern eine gewisse Voreingenommenheit oder Abneigung bewirken könnte, kommt die humoristische Untermalung entgegen. Autor und Zeichner des Büchleins sind ein und dieselbe Person, wobei die Illustrationen dem geschriebenen Text visuelle Stütze leisten, ohne letzteren jedoch zu ersetzen. Darüber hinaus durchziehen zahlreiche kleine Cartoons das Buch, deren Komik unter anderem darin begründet liegt, dass sie Elemente des 20./21. Jahrhunderts (Teleskop, Bücher, Schilder u.a.) in die Welt der historischen Maya einbringen, sowie Sprachspiele und Redewendungen der Gegenwart verwenden.

\footnotetext{
${ }^{6}$ Die Einfügung von Abbildungen des Werks ist in diesem Artikel nicht möglich, frei zugängliche Ausschnitte einer Kindle-Version auf Amazon können aber einen kleinen Einblick geben: <https://www.amazon.com.mx/Losmayas-las-rocas-divertido-ebook/dp/B00MNZU0V4>. (15.11.2017). 


\section{Adressaten und (ideales) Profil der Zieltexte}

Da die Übersetzungen an denselben Verkaufsstellen der Halbinsel erhältlich sind (bzw. waren) wie der AT, ist davon auszugehen, dass diese ebenfalls vorrangig für Touristen gedacht sind, die sich beim Kauf auf Yucatán-Reise befinden. Selbstverständlich handelt es sich bei den deutsch- und englischsprachigen Touristen nicht um eine homogene Gruppe; auch innerhalb der einzelnen Sprachen nicht, denn die Sprecher kommen aus verschiedenen Ländern und Weltteilen, haben unterschiedliche Bildungsvoraussetzungen, haben sich vor ihrer Reise mehr oder weniger intensiv - oder auch gar nicht - mit Mexiko und den Maya auseinandergesetzt und haben unterschiedliche Interessen.

Im Grunde genommen verfolgen AT und ZTe denselben kommunikativen Zweck: Eine leicht verständliche und unterhaltsame Einführung in einige Aspekte der Maya-Kultur zu geben, denn weder die Adressaten des ATs noch die der ZTe sind Maya-Spezialisten. Was letztere allerdings grundsätzlich voneinander unterscheidet, sind ihre völlig unterschiedlichen Vorkenntnisse bezüglich geographischer, geschichtlicher, architektonischer und anderer Mexiko-spezifischer Eigenheiten, sowie der Grad ihrer Vertrautheit mit prähispanischen Bauwerken, mexikanischer Flora, Zutaten der mexikanischen Küche u.s.w. Das erlaubt es, die Adressaten der fremdsprachigen ZTe als gemeinsame Gruppe zu betrachten, ungeachtet dessen, dass diese Gruppe im Inneren sehr heterogen ist.

Die vorgenannten Überlegungen haben gewichtige Konsequenzen für das (ideale) Profil der ZTe. Die starke Unvorhersehbarkeit der jeweiligen Voraussetzungen der Adressaten legen es nahe, die Übersetzungen sprachlich und inhaltlich einem möglichst breit gefächerten Publikum zugänglich zu machen. Sprachlich sollten die ZTe also so gestaltet sein, dass sie ebenfalls von Lesern ab ungefähr 12 Jahren verstanden werden können. Im Vergleich zum AT bleiben als Invarianten, d.h. als Merkmale des ATs (vgl. SCHREIBER 2006: 36) deshalb die Kürze der Absätze erhalten, ebenso der einfache Satzbau, der Gebrauch einer nicht komplexen Standardsprache ohne Fachbegriffe - mit Ausnahme derer, die im Text selbst erklärt und veranschaulicht werden -, die Cartoons können auch umgangssprachliche Wendungen enthalten, um einen mündlichen Sprachgebrauch der Figuren nachzuahmen.

Was allerdings aus unserer Perspektive zwingend dazu führen muss, kleinere inhaltliche Eingriffe vorzunehmen, sind die im Vergleich zur Zielgruppe des ATs unterschiedlichen Voraussetzungen der Adressaten der ZTe, wie vorhergehend ausgeführt. Friedrich 


$$
\text { Gruhn, D.H.; Cabrera, G.R.; Kottenhahn, K. - Touristen auf Yucatán }
$$

Schleiermacher hielt es für unerlässlich, die Übersetzung dem Leser anzunähern, wenn dieser mit der Kultur und dem Umfeld des Originals nicht vertraut ist: "Entweder der Uebersezer lässt den Schriftsteller möglichst in Ruhe, und bewegt den Leser ihm entgegen; oder er lässt den Leser möglichst in Ruhe und bewegt den Schriftsteller ihm entgegen" (Schleiermacher 1963: 47). Zwischen diesen beiden "Methoden", die heute als einbürgernde oder verfremdende Übersetzung bezeichnet werden, sah er keinen Mittelweg.

Im vorliegenden Fall haben wir es zwar nicht mit touristischem Material im engeren Sinne zu tun (wie z.B. Prospekte, Karten, Ortsbeschreibungen u.a.), doch lässt sich das Buch in einen touristischen Kontext eingliedern und dessen Übersetzung sollte wie die der vorgenannten Materialien "ein Werkzeug der sowohl sprachlichen als auch kulturellen Vermittlung” (DURÁN MUÑOZ 2012: 104, Ü. H.G.) sein. Es geht darum, den ausländischen Lesern eine ihnen fremde Welt näher zu bringen. Die Kultur der Maya darf also in der Übersetzung keinesfalls in einen europäischen Kontext umgebürgert werden, etwa indem man den Maya-Göttern eingedeutschte Namen verleihen oder sie gleich gänzlich durch altgermanische Pendants ersetzen würde. Die einschlägige Terminologie und die den Adressaten fremden Inhalte sind zu erhalten, was eine Herausforderung für die Leser der ZTe darstellt. Da diese sich nun in einer Urlaubssituation befinden und das Büchlein eher zufällig mitnehmen, ist ihnen diese Aufgabe zu erleichtern, ansonsten besteht die Gefahr, dass sie es schnell wieder aus der Hand legen. Das bedeutet, dass es durchaus eine Bewegung hin zum Leser geben muss, nämlich indem man ihm gewisse Zusatzinformationen zur Verfügung stellt. Diese können, falls möglich, im Text selbst mit eingebaut oder aber separat in einem Glossar im Anhang ausgeführt werden.

Eine besondere Schwierigkeit der Übersetzung des Buches liegt darüber hinaus darin begründet, dass es sich um eine untergeordnete Übersetzung handelt. Nach Valero Garcés (2000: 77) ist darunter eine Übersetzung zu verstehen, die von außersprachlichen Faktoren (visuellen, typographischen u.a.) abhängt und insofern Einschränkungen unterliegt. Im vorliegenden Fall sind die Einschränkungen von den Zeichnungen vorgegeben - Inhalte und Seiten müssen sich in allen Versionen genau entsprechen, die Übersetzung darf also nicht mehr Raum einnehmen als der AT. Dazu kommt, dass Karten, Zeichnungen und Sprechblasen der Cartoons miteinander und mit dem übrigen Text abgestimmt sein müssen, um eine Störung der Text-Bild-Kohärenz zu vermeiden. 
Nicht zuletzt ist anzumerken, dass die kommunikative Reichweite der ZTe über die des ATs hinausgeht. Es trifft zu, was Durán Muñoz (2012: 112) für touristische Übersetzungen in Spanien ausführt, nämlich den Einfluss dieser Übersetzungen auf das Image eines Landes in seiner Eigenschaft als "Marke". Während sich die Adressaten des ATs nämlich zwischen einer Vielzahl an mexikanischen Publikationen bewegen (können), werden die ZTe für manche Adressaten möglicherweise die einzige mexikanische Veröffentlichung sein, die sie jemals lesen. Deren Qualität verleitet zu Rückschlüssen und Verallgemeinerungen, kann also einen direkten Einfluss auf ihr Mexiko-Bild haben. Diesen Einfluss der ZTe möchten wir in der vorliegenden Arbeit Botschafter-Effekt nennen.

\section{Methodisches Vorgehen}

Bei unserer Analyse stützen wir uns auf ein von Silvia Parra Galiano (2007) vorgestelltes Überprüfungsmodell, das für die Qualitätskontrolle von Übersetzungen in der Berufswelt konzipiert wurde und auf einem funktionalen Übersetzungsverständnis gründet. Oberstes Prinzip ist die genaue Kenntnis des Übersetzungsauftrags, was unter anderem den Übersetzungszweck und das Umreißen der Adressaten beinhaltet (was wir vorhergehend bereits getan haben).

Ein weiteres ihrer Prinzipien besteht darin, den ZT zunächst einmal so zu lesen, als ob man selbst zur Zielgruppe gehöre und die Ausgangssprache nicht verstehe. Dabei ist auf sprachliche und orthographische sowie inhaltlich-logische Aspekte zu achten, ebenso auf das Layout. Erst anschließend wird für die komparative Überprüfung auch der AT hinzugezogen. Parra unterscheidet vier Überprüfungsmodalitäten, die sich mit unterschiedlichen Aspekten auseinandersetzen und, laut der Autorin, auch von unterschiedlichen Experten durchgeführt werden sollten, was wir im Rahmen dieser Arbeit allerdings nicht leisten können. Nachfolgend eine kurze Übersicht der Modalitäten mit den jeweiligen Parametern, die sie umfassen (vgl. ebd.: 213), sowie eine stichwortartige Beschreibung der einzelnen Parameter (vgl. ebd.: 204207), wobei der Einbezug eines Übersetzungsprofils von uns stammt. 
Gruhn, D.H.; Cabrera, G.R.; Kottenhahn, K. - Touristen auf Yucatán

\begin{tabular}{|c|c|c|}
\hline $\begin{array}{l}\text { Überprüfungs- } \\
\text { modalität }\end{array}$ & Parameter & Beschreibung \\
\hline \multirow[t]{3}{*}{ Inhaltliche } & - Logik & $\begin{array}{l}\text { Textaufbau, Kohärenz und Kohäsion } \\
\text { des ZTs, Ideenführung ohne Wider- } \\
\text { sprüche oder Sinnlosigkeiten }\end{array}$ \\
\hline & - Daten & $\begin{array}{l}\text { faktische, konzeptuelle, numerische } \\
\text { u.ä. Fehler }\end{array}$ \\
\hline & - Fachsprache & $\begin{array}{l}\text { lexikalisch, syntaktisch, rhetorisch der } \\
\text { Zielsprache und dem Profil des ZTs } \\
\text { angemessen }\end{array}$ \\
\hline \multirow[t]{3}{*}{ Linguistische } & - Fachsprache & $\begin{array}{lll}\text { linguistische Überprüfung } & \text { von } \\
\text { Terminologie und Phraseologie } & \end{array}$ \\
\hline & $\begin{array}{l}\text { - Normen und Gebrauch der } \\
\text { Zielsprache }\end{array}$ & $\begin{array}{l}\text { Anwendung des gültigen linguis- } \\
\text { tischen Codes der Zielsprache in } \\
\text { Bezug auf Grammatik, Syntax, } \\
\text { Orthographie, Lexik usw., Klarheit der } \\
\text { Sätze, Stil, Register. Mit besonderem } \\
\text { Augenmerk auf etwaige Interferenzen } \\
\text { aus der Ausgangssprache. }\end{array}$ \\
\hline & - Anpassung an die Adressaten & 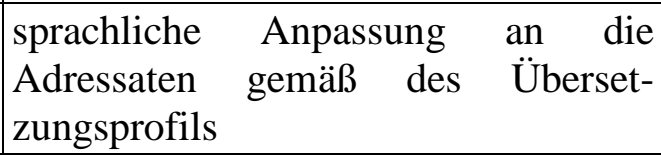 \\
\hline \multirow[t]{3}{*}{ Funktionelle } & - $\quad$ Anpassung an die Adressaten & $\begin{array}{l}\text { (Siehe dazu unsere Ausführungen im } \\
\text { Anschluss an diese Tabelle.) }\end{array}$ \\
\hline & - Genauigkeit & \begin{tabular}{|lll}
\multicolumn{3}{l}{ Präzision der Übertragung der im AT } \\
enthaltenen Nachricht & gemäß & des \\
Übersetzungsprofils & (d.h. & des \\
Übersetzungszwecks & und & der \\
Bedürfnisse der Adressaten) & \\
\end{tabular} \\
\hline & - $\quad$ Vollständigkeit & $\begin{array}{l}\text { Vollständige Übertragung der Nach- } \\
\text { richt des ATs ohne unnötige (d.h. sich } \\
\text { nicht aus dem Profil des ZTs erge- } \\
\text { bende oder anderweitig begründbare) } \\
\text { Einfügungen oder Auslassungen. }\end{array}$ \\
\hline \multirow[t]{2}{*}{ Üb. des Layouts } & - Vollständigkeit & $\begin{array}{l}\text { Unabsichtliche } \begin{array}{l}\text { Auslassungen } \\
\text { Seite, eines } \\
\text { Abschnitts, }\end{array} \\
\text { Illustration u.s.w. }\end{array}$ \\
\hline & $\begin{array}{l}\text { - } \begin{array}{l}\text { Gestaltung und Orthotypogra- } \\
\text { phie }\end{array} \\
\end{array}$ & $\begin{array}{l}\text { Anordnung und Gestaltung muss } \\
\text { übersichtlich sein und darf nicht zu } \\
\text { Unklarheiten führen, vollständige } \\
\text { Nummernführung (Inhaltsverzeichnis, } \\
\text { Seiten, Tabellen u.a.), Ränder, } \\
\text { Zeilenabstand u.s.w., zielsprachen- } \\
\text { gerechte Anwendung von } \\
\text { typographischen Elementen (Groß- } \\
\text { und Kleinschreibung u.a.). }\end{array}$ \\
\hline
\end{tabular}


Gruhn, D.H.; Cabrera, G.R.; Kottenhahn, K. - Touristen auf Yucatán

Im Unterschied zu Parra sehen wir die Anpassung an den Adressaten nicht als einen mehrerer Unterparameter an. Zwar lassen sich einzelne Teilaspekte getrennt betrachten, wie die sprachliche Anpassung des ZTs an die Adressaten, doch eine Gesamtbewertung dieser Anpassung steht in Zusammenhang mit allen übrigen Parametern und ergibt sich aus dem Gesamtübersetzungsprofil. Eine an die Adressaten und den kommunikativen Zweck angepasste Übersetzung kann als angemessen bezeichnet werden und deckt insofern dasselbe Konzept ab wie die von uns verfolgte Adäquatheit der Translate. Diese ergibt sich folglich als übergeordnetes Kriterium aus der Bewertung sämtlicher Einzelparameter.

\section{Die einsprachige Überprüfuns}

In einem ersten Durchgang werden sämtliche Überprüfungsparameter aufgegriffen, die nicht des Vergleichs mit dem AT bedürfen. Das ist vor allem die allgemeine Verständlichkeit, die mit der inhaltlichen Kohäsion und Kohärenz zusammengeht, des Weiteren der normsprachige Gebrauch, die Stilebene und einige orthotypographische Elemente. Textbeispiele sind in Tabellenform aufgeführt, die Nummern in der linken Spalte beziehen sich immer auf die Buchausgaben in allen drei Sprachen (Spanisch, Deutsch, Englisch), da alle eine identische Seiteneinteilung aufweisen.

\section{1 Sprache}

Unser Übersetzungsprofil sieht den Gebrauch einer nicht komplexen Standardsprache vor. Im Gegensatz zum englischen ZT, der nur in Ausnahmefällen Abweichungen von der Normsprache aufweist, sind die Normverstöße der deutschsprachigen Version sehr zahlreich, Rechtschreib-, Grammatik-, Wortbildungs- oder Wortwahlfehler durchziehen den Text. Nachfolgend eine (unvollständige) Auswahl:

\begin{tabular}{|l|l|}
\hline Seite & \multicolumn{1}{c|}{ Deutschsprachiger ZT } \\
\hline & Rechtschreibung \\
\hline 18 & Intelektuelle (statt: intellektuelle) \\
\hline 23 & gemeinsan (statt: gemeinsam) \\
\hline 35 & bleibts (statt: bleibt's) \\
\hline 52 & Hunab-Ku's Sohn (statt Hunab-Kus Sohn) \\
\hline
\end{tabular}


Gruhn, D.H.; Cabrera, G.R.; Kottenhahn, K. - Touristen auf Yucatán

\begin{tabular}{|l|l|}
\hline 88 & $\begin{array}{l}\text { Cronik (statt: Chronik) } \\
\text { Mythus (statt: Mythos) }\end{array}$ \\
\hline 106 & tatuierten (statt: tätowierten) \\
\hline 109 & Profet (statt: Prophet) \\
\hline & $\begin{array}{l}\text { Grammatik (Präpositionen, Konjugationen, morphologische Aspekte, Satzbau } \\
\text { u.a.) }\end{array}$ \\
\hline 9 & man wurde auf der Region sesshaft (statt: in der Region) \\
\hline 10 & $\begin{array}{l}\text { auf einem Gebiet mit von 400,000 Quadratkilometern (statt: in einem 400.000 } \\
\text { Quadratkilometer großen Gebiet) }\end{array}$ \\
\hline 19 & ich hab's... gesagt, das du... (statt: , dass du...) \\
\hline 29 & $\begin{array}{l}\text { Das ist klar: Bei den Mayas... (statt: Klar ist, dass... An dieser Stelle ist kein } \\
\text { Rückbezug vorhanden) }\end{array}$ \\
\hline 48 & Der erste Vater erweckte ... zum Leben (statt: erwachte) \\
\hline 83 & Die Maya Gewölbe-Stil (statt: der) \\
\hline 87 & $\begin{array}{l}\text {...welche Männer in verschiedenen gesellschaftlichen Positionen darstellten. (statt: } \\
\text { Männer aus verschiedenen gesellschaftlichen Klassen) }\end{array}$ \\
\hline 89 & ...handelten sie über (statt: von) \\
\hline 106 & Dazu wurden vor dem Kind Harzkugeln aufgehangen (statt: aufgehängt) \\
\hline
\end{tabular}

Die deutschsprachige Version fällt besonders durch ihre unsichere Wortwahl auf, wiederholt werden feste Wortverbindungen nicht normsprachengerecht verwendet, die lexikalische Kohäsion ist gestört bzw. der Stil wirkt unbeholfen oder führt zu Unklarheiten:

\begin{tabular}{|l|l|}
\hline Seite & \multicolumn{1}{c|}{ Deutschsprachiger ZT } \\
\hline 11 & $\begin{array}{l}\text { Phraseologie, lexikalische Kohäsion } \\
\text { Der Ausgangspunkt befindet sich in einem Gebiet... und zieht sich... bis in den } \\
\text { deshalb nicht ziehen) }\end{array}$ \\
\hline 15 & kristallisierten sich zwei Typen von Klassen heraus (Stil) \\
\hline 27 & $\begin{array}{l}\text { Die Gesellschaft der Mayas stimmte mit ihrer religiösen Weltanschauung überein. } \\
\text { (Stil) }\end{array}$ \\
\hline 35 & Geschäfte abhandeln (statt: aushandeln, abwickeln) \\
\hline 50 & $\begin{array}{l}\text { Die Mayas dachten, dass die Götter das Weltall schufen, um ihre Existenz durch } \\
\text { den Menschen zu unterhalten. (Stil) }\end{array}$ \\
\hline 51 & $\begin{array}{l}\text { Fast alle Götter waren gnädig..., wenn sie den Mayas ein Leben ohne } \\
\text { Überstürzungen erteilten. (Stil) }\end{array}$ \\
\hline 53 & $\begin{array}{l}\text {...integrierte sich ein unter dem Namen Kukulcan... in die Kultur der Mayas ein. } \\
\text { (gemeint ist wohl "gliederte sich ein" oder einfach nur “integrierte sich in") }\end{array}$ \\
\hline
\end{tabular}


Gruhn, D.H.; Cabrera, G.R.; Kottenhahn, K. - Touristen auf Yucatán

\begin{tabular}{|l|l|}
\hline 61 & $\begin{array}{l}\text { Die Plantagenbesitzer organisierten Feste, um den Schutz ihrer Pflanzungen } \\
\text { anzubeten. } \\
\text { (angebetet wurde der Heilige Kakao-Gott und nicht der Schutz) }\end{array}$ \\
\hline 104 & $\begin{array}{l}\text {...unterschieden zwischen begangenen vorsätzlichen Straftaten... (statt: vorsätzlich } \\
\text { begangenen Straftaten... }\end{array}$ \\
\hline 105 & $\begin{array}{l}\text { Diebe wurden dazu gezwungen, das Geraubte wieder zurückzugeben. (Diebe } \\
\text { stehlen, sie rauben nicht) }\end{array}$ \\
\hline
\end{tabular}

Auch in der Version auf Englisch lassen sich einige ungewöhnliche Wendungen und Satzkonstruktionen finden, deren Grund wir später noch erläutern werden:

\begin{tabular}{|l|l|}
\hline Seite & \multicolumn{1}{|c|}{ Englischsprachiger ZT } \\
\hline 10 & $\begin{array}{l}\text {.. the Usumacinta and Motagua rivers are born in its mountains (statt: have their } \\
\text { sources) }\end{array}$ \\
\hline 41 & the priests suffered nicely \\
\hline 46 & Angry, they cut off his head... \\
\hline
\end{tabular}

\subsection{Inhalt}

Die Trennung in inhaltliche und linguistische Überprüfungsparameter ist in Wirklichkeit eine künstliche, verhält es sich doch so, dass sprachliche Unklarheiten häufig auch inhaltliche nach sich ziehen. Logik und inhaltlicher Zusammenhang sind in der deutschsprachigen Version an mehreren Stellen empfindlich gestört. Unter anderem enthält sie falsche Zahlenangaben, die selbst ein Laie sofort erkennen kann: Auf Seite 20 werden die Maya ins 19. Jahrhundert versetzt (statt ins 9.) und das Vigesimalsystem wird zum Zehnersystem (S. 77), was in Widerspruch zu den übrigen Ausführungen des Kapitels steht.

Bereits der Titel des deutschen ZTs wirft Fragen auf: Lag die Blütezeit der Maya denn in der Steinzeit? Und wie ist es möglich, dass das Maya-Gebiet Länder wie Mexiko, Guatemala, Belize u.a. einschloss - gab es die denn auch schon in der Steinzeit? (S. 10). Andere Begriffe klingen widerum sehr gegenwärtig, wie z.B. der Taufname (S. 108) - waren denn auch die prähispanischen Maya getauft? Anzumerken ist, dass die Verfremdung durch Elemente der Gegenwart im Original nur in den Zeichnungen und Cartoons auftritt, nicht aber im Erklärteil.

Der englischsprachige Zieltext enthält auch einige Ambiguitäten, so z.B. wenn maíz einmal mit maize und dann wieder mit corn übersetzt wird. Der Leser muss sich also fragen, ob es sich hierbei um ein- und dieselbe Pflanze handelt, oder ob die Maya vielleicht schon den 


$$
\text { Gruhn, D.H.; Cabrera, G.R.; Kottenhahn, K. - Touristen auf Yucatán }
$$

Weizen kannten. Und obgleich die Himmelsrichtungen für die Maya äußerst bedeutsam waren, so kannten sie doch noch keinen compass (ebenfalls im Erklärteil, S. 49), passender wäre gewesen, von cardinal directions zu sprechen.

Weitere inhaltliche Auffälligkeiten bei der einsprachigen Lektüre sind, dass manche Cartoons (im Deutschen wie im Englischen) wenig Sinn ergeben oder einfach nicht witzig sind, so z.B. Nothin to fry in the Maya - Pan! (S. 24). Und sowohl der deutsch- als auch der englischsprachige Adressat werden über Begriffe stolpern, mit denen sie wahrscheinlich nicht viel anfangen können, und die in den ZTen nicht erklärt werden: "Mexiken", "Kazikentum", "Saramujo" - "ball games", "anona".

Auf die beiden letzteren Punkte werden wir in einem späteren Kapitel noch einmal zurückkommen.

\subsection{Layout und Orthotypographie}

Alle Versionen sind ausschließlich in Großbuchstaben geschrieben. Im deutschen Zieltext sind die Umlaute korrekt gesetzt, allerdings existiert kein “ $\beta$ ”, was wohl computertechnische Gründe hat. In beiden Versionen gibt es keine einheitliche Zifferngruppierung (teils mit Punkt, teils mit Komma, teilweise ohne Gliederungszeichen). Die Akzente, die im Spanischen auch bei Wörtern in der Maya-Sprache benutzt werden, sind herausgenommen, allerdings nicht überall (so wurden sie z.B. sowohl im Deutschen als auch im Englischen auf den Seiten 71 und 109 stehen gelassen, aber auch bei den Namen auf einigen Karten). Auffällig ist, dass in beiden ZTen die Karten manchmal ganz (z.B. auf S. 82), manchmal teilweise (z.B. auf S. 18) und manchmal überhaupt nicht übersetzt wurden (z.B. auf S. 10/11). Im Übrigen wurden die Karten auch nicht an das Vorverständnis der Adressaten angepasst: Ebenso wie im AT reicht das MayaGebiet auf Seite 8 der ZTe bis nach Texas und Florida, und wer nicht ohnehin schon weiß, wo Tikal oder Copán liegen, wird Schwierigkeiten haben, sie auf den Karten auf Seite 17 oder 18 zu finden. Insgesamt entsteht der Eindruck, dass dem Layout keine besondere Aufmerksamkeit gewidmet wurde. 


\subsection{Fazit der einsprachigen Lektüre}

Wie wirken nun die ZTe auf ihre jeweiligen Adressaten? Die deutschsprachigen Leser werden den ZT zwar über weite Teile verstehen, bemerken aber neben einigen orthotypographischen Schwächen, dass die Beherrschung der deutschen Schriftsprache unsicher und standardsprachigen Normen an vielen Stellen nicht angemessen ist. Darüber hinaus werden sie auf mehrere inhaltlich unklare Stellen stoßen und sogar offensichtlich falsche Informationen finden. Sie werden mit unbekannten Begriffen und Konzepten konfrontiert sein, bei einigen Cartoons den Witz nicht verstehen und geographische Angaben nicht immer nachvollziehen können.

Die Adressaten des englischsprachigen ZTs werden nur vereinzelt Normverstöße und unklare Stellen vorfinden, doch auch sie werden auf unbekannte Begriffe und Konzepte stoßen und geographische Angaben nicht immer nachvollziehen können.

\section{Die vergleichende Überprüfung}

In einem zweiten Durchgang werden nun AT und ZTe detailliert verglichen. Das umfasst zunächst einmal alle Parameter, die im vorigen Durchgang nicht aufgegriffen wurden, darüber hinaus aber auch die Abgleichung von bereits aufgefundenen sprachlich unklaren Stellen; möglicherweise gibt der Vergleich Aufschluss über die Fehlerquellen.

\subsection{Vollständigkeit}

Der Ausgangstext ist im Deutschen wie im Englischen mit Einschränkungen vollständig übernommen worden. Die Seitenzahlen entsprechen sich in allen Versionen. In beiden ZTen wurden jeweils zwei Sätze ausgelassen (jeweils die Erklärung des Maya-Konzeptes “sacbé” auf S. 35, sowie im Deutschen auf S. 14 unten und im Englischen auf S. 10 unten), ohne dass ein triftiger Grund für die Auslassungen zu erkennen wäre.

Eine gewichtige Auslassung weist der deutsche ZT auf Seite 11 auf: Vergessen wurde nur ein einziges Wort, nämlich die Region "Petén", bei der Beschreibung des Zentralen Siedlungsgebiets der Maya. Nun ist zu verstehen, dass dieses Gebiet ganz Guatemala umfasst. Dabei entsteht ein Widerspruch zur Beschreibung des südlichen Gebiets, wo ein Teil 
Gruhn, D.H.; Cabrera, G.R.; Kottenhahn, K. - Touristen auf Yucatán

Guatemalas noch einmal aufgeführt ist. Auch stimmen Beschreibung und Markierung auf der Karte nun nicht mehr überein.

\subsection{Sprache}

Die zweisprachige Überprüfung bringt weitere, auf Sprachfehler zurückgehende Übertragungsfehler zutage, wobei wir es bei zwei Beispielen belassen wollen:

\begin{tabular}{|l|l|l|}
\hline 27 & $\begin{array}{l}\text {.. arriba estaban los de arriba y los } \\
\text { de más arriba }\end{array}$ & der obrigen und höherstellten Herrschaft. \\
\hline & $\begin{array}{l}\text { Erst der Vergleich macht deutlich, dass es hier nicht um ein und dieselbe Herrschaft } \\
\text { geht, sondern um verschiedene Personengruppen, von denen eine noch über der } \\
\text { anderen stand. }\end{array}$ \\
\hline 105 & $\begin{array}{l}\text { Por deudas no apresaban a nadie } \\
\text { (nadie fiaba). }\end{array}$ & $\begin{array}{l}\text { Im Falle von Schulden wurde niemand } \\
\text { verurteilt (keiner erteilte Kredit). }\end{array}$ \\
\hline & $\begin{array}{l}\text { Wenn es keine Kredite gab, konnte doch auch niemand Schulden haben? } \\
\text { Bereits die einsprachige Lektüre wirft Fragen auf. Doch erst die zweisprachige } \\
\text { klärt das Problem: Die Präposition ist falsch übersetzt, es muss "wegen" heißen. }\end{array}$ \\
\hline
\end{tabular}

Und erst die zweisprachige Überprüfung wirft Licht auf die Quelle mancher ungewöhnlichen Wendungen und Satzkonstruktionen, vor allem in der englischen Version: es handelt sich um Interferenzen aus dem Spanischen. Kupsch-Losereit (2004: 543) erklärt den Begriff folgendermaßen:

Die moderne Translatologie gebraucht den Terminus Interferenz für die Projektion von Merkmalen des AT in den ZT, deren Ergebnis eine Verletzung der ZT-Normen und / oder ZTKonventionen ist. [...] Auch in grammatisch und lexikalisch völlig akzeptablen Sätzen gibt es Interferenzen, weil sich die tatsächliche Verwendung und die kommunikative Wirkung der grammatischen, thematisch-inhaltlichen, mikro- und makrotextuellen Strukturen sowie die jeweiligen funktionellen Sprachen in AT und ZT unterscheiden.

Die Gegenüberstellung einiger spanisch- und englischsprachigen Stellen lässt deutlich die (unbewusste) Übernahme sprachlicher Strukturen des ATs im englischen ZT erkennen:

\begin{tabular}{|l|l|l|}
\hline Seite & \multicolumn{1}{|c|}{ AT } & \multicolumn{1}{c|}{ Englischsprachiger ZT } \\
\hline 10 & $\begin{array}{l}\text {..en sus montañas nacen los ríos } \\
\text { Usumacinta y Motagua. }\end{array}$ & $\begin{array}{l}\text {.. the Usumacinta and Motagua rivers } \\
\text { are born in its mountains. }\end{array}$ \\
\hline 41 & ...los sacerdotes sufrían de lo lindo... & ...the priests suffered nicely... \\
\hline 46 & Enojados, le cortaron la cabeza... & Angry, they cut off his head... \\
\hline
\end{tabular}


Übernommen wurde in beiden ZTen die einfache Syntax (kurze Absätze mit nicht mehr als zwei oder drei kurzen Sätzen), die wir oben als Invariante des Übersetzungsprofils ausgemacht haben. Doch auch dieser Sachverhalt ist als Interferenz zu interpretieren und nicht etwa als Ergebnis eines Übersetzungsplans.

\subsection{Fachbegriffe aus Kultur und Natur}

Der AT enthält zahlreiche Begriffe, die mit der Maya-Kultur, dem Kulturraum Mexiko allgemein oder der mexikanischen Pflanzenwelt in Zusammenhang stehen. Dabei sind für den Übersetzer drei Fälle zu unterscheiden:

- Viele Konzepte (vor allem auf Maya) werden im AT selbst erklärt und sind Thema der Ausführungen. Die Begriffe werden in den ZTen auf Maya beibehalten.

- Einige Mexiko-spezifische Kulturelemente können auch bei den Adressaten als bekannt vorausgesetzt und somit in der Sprache des ATs beibehalten werden (z.B. Tortillas).

- Weitere Konzepte werden vielen Adressaten der ZTe - im Gegensatz zu den mexikanischen Lesern - nicht vertraut sein, bzw. sie sind bei ihrem Besuch auf Yucatán eben zum ersten Mal damit in Berührung gekommen (z.B. juego de pelota, cenote, mexicas, quetzal, Popol $V u$ u.a.).

Letztere bedürfen einer besonderen Behandlung in den ZTen. Im deutschen ZT wurde z.B. das Wort "Mexiken" (S. 53) für mexicas erfunden, bei dem sicher kaum jemandem klar ist, welche Gruppe damit gemeint ist; analog dazu heißt es im Englischen "mexicas". Das ist zwar ein existierender Fachterminus, doch das viel bekanntere "Azteken" bzw. "aztecs" wäre für die Adressaten beider Sprachen eindeutiger gewesen. Das deutsche "Kazikentum" (S. 25) ist ebenfalls ein Fachterminus, der hätte ersetzt oder erklärt werden können; das englische "chieftaincies" wirkt im Vergleich dazu aufschlussreicher.

Andere terminologische Fehlgriffe hätten vermieden werden können, wenn die Übersetzer Fachtexte zu Rate gezogen hätten (nicht umsonst gehört die Recherchefähigkeit zu den Grundkompetenzen eines Übersetzer). So wird z.B. aus einem curandero (S. 31) ein "Quacksalber" - diese negative Konnotation umfasst der spanische Begriff nicht (im 
Gruhn, D.H.; Cabrera, G.R.; Kottenhahn, K. - Touristen auf Yucatán

Englischen wurde das neutralere "healer" gewählt) -, und autosacrificio (S. 43) wird mit "Selbstopferung” übersetzt, es handelte sich aber nicht um eine Selbsttötung, sondern um eine Selbstkasteiung (vgl. PREM 2011: 57).

Auch wurde die Terminologie der historischen Maya-Perioden im Deutschen nicht recherchiert (S. 13). So erscheint z.B. der Begriff "Spätklassik" als Oberbegriff einer Periode und als Unterklasse einer anderen, und auch sonst wirkt die Einteilung terminologisch konfus. Aufschluss hätte der Maya-Experte Berthold Riese (2011) geben können.

Mexikanische Kulturpflanzen (vgl. S. 97 u. 99) wurden zumeist einfach auf Spanisch übernommen, teilweise mit dem Versuch einer orthographischen Eindeutschung: Anona, Pflaumen, Papaja, Nance, Chicozapote und Saramujo (statt der deutschen Rechtschreibung Papaya und Saramuyo). Ob der Adressat mit diesen oder deutschen Pflanzennamen wie "Wollbaum", "Mispelbaum", “Ceiba” oder "Sapote-Frucht” etwas anfangen kann, bleibt ihm ganz allein überlassen. Ähnlich verhält es sich im Englischen.

Da es nicht immer möglich ist, unbekannte Konzepte in einer dem Adressaten bekannten oder unmittelbar verständlichen Terminologie auszudrücken und da die räumlichen Einschränkungen weitere Erklärungen im Textteil nur selten erlauben, wäre auf jeden Fall in beiden ZTen ein kleines Glossar am Ende angebracht gewesen (im Falle der Pflanzen evtl. auch bebildert). Nur so hätte eine Annäherung an den Leser gewährleistet werden können.

\subsection{Genauigkeit der Übertragung}

Wir interessieren uns hier vor allem für die inhaltlich-semantische Entsprechung zwischen AT und ZT (denn gegenüber dem AT-Sender - oder genauer gesagt dem AT - haben wir uns ja zur Loyalität verpflichtet) und untersuchen, inwieweit diese inhaltlich übereinstimmen (wobei im Hinblick auf die Zielgruppe natürlich bewusste Abweichungen nötig sein können). Diese Loyalität schließt weitere Überprüfungsparameter, die wir in anderen Kapiteln behandeln, wie Vollständigkeit, Inhalt, Lexik oder Zusammenspiel von Text und Bild allerdings ein. Nicht begründbare Auslassungen, falsche Datenübernahme, ein unklarer Satzbau oder der Verlust des Zusammenhangs zwischen Text und Bild stellen immer schon Loyalitätsverstöße am AT dar. In diesem Abschnitt nehmen wir daher lediglich einige Störungen des Sinnzusammenhangs auf, die bei der einsprachigen Lektüre in der Zielsprache nicht unmittelbar auffallen, da die Sätze logisch und grammatisch korrekt abgefasst sind. Die Ursachen liegen häufig im Nichterkennen grammatischer Strukturen oder einzelner Wörter des ATs.

Pandaemonium, São Paulo, v. 21, n. 35, set.-dez. 2018, p. 24-46 
Gruhn, D.H.; Cabrera, G.R.; Kottenhahn, K. - Touristen auf Yucatán

\begin{tabular}{|c|c|c|}
\hline Seite & Spanisch & Deutsch \\
\hline \multicolumn{3}{|c|}{ GRAMMATIK } \\
\hline \multirow[t]{2}{*}{18} & $\begin{array}{l}\text {...con el uso de terrazas y canales de } \\
\text { riego }\end{array}$ & $\begin{array}{l}\text { Die Landwirtschaft entwickelte sich dank } \\
\text { der Anlage von Bewässerungskanälen und } \\
\text { Terrassen... }\end{array}$ \\
\hline & \multicolumn{2}{|c|}{$\begin{array}{l}\text { "Riego" bezieht sich sowohl auf die Terassen als auch auf die Kanäle. Dieser } \\
\text { Aspekt verschwindet im Deutschen. }\end{array}$} \\
\hline \multirow[t]{2}{*}{73} & $\begin{array}{l}\text { Cuando el sol se oscurecía en pleno } \\
\text { día, el pueblo entraba en pánico. }\end{array}$ & $\begin{array}{l}\text { Als sich dann die Sonne mitten am Tag } \\
\text { verdunkelte, geriet das Volk in Panik. }\end{array}$ \\
\hline & \multicolumn{2}{|c|}{ Zeit falsch interpretiert: Es war ein Vorgang, der sich wiederholte. Wenn... } \\
\hline \multicolumn{3}{|c|}{ WORTEBENE } \\
\hline \multirow[t]{2}{*}{25} & $\begin{array}{l}\text {... cuando de repente aparecieron } \\
\text { ellos, barbados y temperamentales: los } \\
\text { españoles del viejo mundo }\end{array}$ & $\begin{array}{l}\text {... als jene Barbaren und } \\
\text { temperamentvollen Spanier vom alten } \\
\text { Kontinent.... }\end{array}$ \\
\hline & \multicolumn{2}{|c|}{$\begin{array}{l}\text { Der Fehler scheint auf das Original zurückzugehen, wo es "barbudos" heißen } \\
\text { müsste. Die Spanier waren also bärtig und temperamentvoll (wie im englischen ZT } \\
\text { korrekt erkannt wurde). }\end{array}$} \\
\hline \multirow[t]{2}{*}{37} & hombres inferiores & niedrige Männer \\
\hline & \multicolumn{2}{|c|}{$\begin{array}{l}\text { Statt: einfaches Volk, untere Schichten. Anzunehmen ist, dass auch Frauen } \\
\text { dazugehörten. }\end{array}$} \\
\hline \multicolumn{3}{|c|}{ SATZEBENE } \\
\hline \multirow[t]{2}{*}{27} & $\begin{array}{l}\text { Los sacerdotes descendientes de los } \\
\text { mismísimos dioses... }\end{array}$ & $\begin{array}{l}\text { Die Priester als direkte Nachfolger der } \\
\text { Gottheiten regierten und bestimmten. }\end{array}$ \\
\hline & \multicolumn{2}{|c|}{$\begin{array}{l}\text { Hat im Original etwas Ironisches (Abkömmlinge der Götter selbst), das im } \\
\text { Deutschen verschwindet. }\end{array}$} \\
\hline
\end{tabular}

Auch in der englischsprachigen Version lassen sich einige Beispiele vor allem semantischer Natur finden:

\begin{tabular}{|l|l|l|}
\hline Seite & Spanisch & Englisch \\
\hline 9 & $\begin{array}{l}\text { Y los pueblos de Mesoamérica tenían } \\
\text { una cultura uniforme. }\end{array}$ & $\begin{array}{l}\text { And the towns of Mesoamerica had a } \\
\text { uniform culture. }\end{array}$ \\
\hline & $\begin{array}{l}\text { Es handelt sich hier nicht um Städte, sondern um Völker (peoples), in der dazu- } \\
\text { gehörigen Karte aus dem Jahr 2000 v.Chr. ist keine einzige Stadt eingezeichnet. }\end{array}$ \\
\hline 35 & $\begin{array}{l}\text {..lo más preciado para los mayas: el } \\
\text { cacao, la moneda... }\end{array}$ & $\begin{array}{l}\text {..obtaining what was most sacred to the } \\
\text { Maya: cacao beans }\end{array}$ \\
\hline & $\begin{array}{l}\text { Die Kakaobohne war bei den Maya zwar tatsächlich heilig, hier geht es aber um } \\
\text { materiellen Wert. Die englische Version bringt ein ironisches Moment in den Text } \\
\text { ein. Valuable oder precious wären näher am Ausgangstext geblieben. }\end{array}$ \\
\hline
\end{tabular}


Gruhn, D.H.; Cabrera, G.R.; Kottenhahn, K. - Touristen auf Yucatán

\begin{tabular}{|l|l|l|}
\hline 56 & $\begin{array}{l}\text {...donde llegaban los espíritus de casi } \\
\text { todos los hombres cuando morían. }\end{array}$ & $\begin{array}{l}\text {..where the spirits of all the men arrived } \\
\text { when they died. }\end{array}$ \\
\hline 58 & Nicht alle kamen da hin, nur fast alle. \\
\hline & $\begin{array}{l}\text { Ixchel se asocia con el agua de los } \\
\text { lagos y las fuentes. }\end{array}$ & $\begin{array}{l}\text { Ixchel is associated with the water of lakes } \\
\text { and fountains. }\end{array}$ \\
\hline & $\begin{array}{l}\text { Springs oder sources (Quellen) wäre hier besser gewesen, denn es geht nicht um } \\
\text { Springbrunnen, sondern um Fortpflanzung und Geburt (Ixchel ist die dafür } \\
\text { zuständige Göttin) }\end{array}$ \\
\hline
\end{tabular}

\subsection{Die Cartoons in der Übersetzung}

Sämtliche Zeichnungen wurden in der Übersetzung ohne jede Veränderung übernommen. Der Witz der Cartoons liegt häufig darin begründet, dass Episoden mit Elementen der Gegenwart angereichert sind. Dadurch entsteht eine Art Paralleldiskurs, so z.B. wenn heutige Verkaufsmethoden (ein Schild mit dem Wortlaut "Heute großer Ausverkauf", S. 34) in die Welt der Maya übertragen werden, wenn die Kakaobohne eine Fälschung ist, weil "Made in China" drauf steht (S. 35), oder der Astronom von modernen Teleskopen überfordert ist (S. 72).

Neben grammatischen und lexikalischen Verstößen, wie sie auch im Übrigen deutschsprachigen ZT auftreten, ist stellenweise eine unzureichende Abgleichung mit den Bildern auszumachen, was zu einer Störung der Kohärenz Text-Bild führt. Das scheint weniger auf Raumprobleme zurückzuführen sein, wie man bei solch einer untergeordneten Übersetzung erwarten könnte, sondern darauf, dass beim Übersetzen vor allem auf den Schrifttext der Sprechblasen geachtet wurde, und die übrigen Textelemente dabei vernachlässigt wurden.

Nachfolgend einige Beispiele (der deutschen Version): Im Cartoon auf Seite 33 wird ein Steuerflüchtiger lexikalisch mit Flüchtling verwechselt, der Zusammenhang zwischen Flüchtling und der vorhergehenden Ausführung zu Tributzahlungen ist nun schwerer $\mathrm{zu}$ durchschauen. Im Cartoon auf Seite 43 wird Klatsch mit Quatsch (Unsinn) verwechselt, der “quatschhafteste Priester" sollte eher “das größte Klatschmaul” oder “die größte Plaudertasche” heißen. Denn Quatsch machen und das Zerstechen der Zunge stehen in keinem Zusammenhang mehr, auch wenn noch nachzuvollziehen ist, was gemeint war.

Im Cartoon auf Seite 69 wird ein Maya nach einem Gelage auf einer Bahre weggetragen und plant, das mit fünf Fastentagen abzubüßen. Das Wortspiel des ATs greift auf die Antonyme "zahlen" und "schulden" zurück: "Pago y me quedan debiendo" (ich zahle das ab und man wird 


\section{Gruhn, D.H.; Cabrera, G.R.; Kottenhahn, K. - Touristen auf Yucatán}

mir noch schulden). "Büßen” und "schulden", wie es in der deutschen Übersetzung lautet, sind dagegen keine Antonyme, was der komischen Situation ihre Klarheit nimmt.

Im Cartoon auf Seite 98 ist ein Maya gerade dabei, zu seiner Frau in die Hängematte zu steigen und erklärt ihr, eben drei Kilo Salz verspeist zu haben. Was das bedeutet, erfahren wir in der spanischsprachigen Version, denn dort heißt es, dass Salz sogar als Stärkungs- sprich Potenzmittel verwendet wurde. In der deutschen Ausgabe steht dagegen lediglich, dass Salz auch zum Einreiben verwendet wurde, der Witz geht damit vollständig verloren.

Im englischsprachigen ZT konnten wir keine vergleichbaren Probleme entdecken.

\subsection{Redewendungen}

Das Nachahmen einer mündlichen Sprechweise bewirkt, dass insbesondere die Cartoons (aber nicht ausschließlich diese) im AT Redewendungen oder andere Formen metaphorischen Sprechens enthalten. Diese wurden im deutschen ZT mehrmals nicht erkannt bzw. als

Denotation übersetzt. Nachfolgend einige Beispiele mit Erläuterungen:

\begin{tabular}{|c|c|c|}
\hline Seite & Originalversion & Übersetzung ins Deutsche \\
\hline \multirow[t]{2}{*}{18} & $\begin{array}{l}\text { Los grandes logros intelectuales }[\ldots] \\
\text { se volvieron armas de poder. }\end{array}$ & $\begin{array}{l}\text { Zu den wichtigsten Waffen für die } \\
\text { Machtausübung zählten vor allem } \\
\text { intelektuelle [sic] Errungenschaften wie... }\end{array}$ \\
\hline & \multicolumn{2}{|c|}{$\begin{array}{l}\text { Es handelt sich nicht um eine Aufzählung von Waffen, sondern um einen } \\
\text { Entwicklungsprozess, bei dem Errungenschaften zu Machtfaktoren wurden. }\end{array}$} \\
\hline \multirow[t]{2}{*}{24} & $\begin{array}{l}\text {..la ciudad de Mayapán fue borrada } \\
\text { del mapa. }\end{array}$ & $\begin{array}{l}\text { Mayapan war plötzlich nicht mehr auf der } \\
\text { Landkarte. }\end{array}$ \\
\hline & \multicolumn{2}{|c|}{$\begin{array}{l}\text { Die im Spanischen gängige und vielseitig einsetzbare Redewendung ("von der } \\
\text { Landkarte getilgt werden") ist im Deutschen als Metapher verständlich. Im hiesigen } \\
\text { Kontext geht es allerdings nicht um ein Verschwinden, sondern um ein aktives } \\
\text { Ausgelöscht werden. Aus der Redewendung herausgelöst wirkt das Wort } \\
\text { "Landkarte" im Deutschen anachronisch, da die Maya keine Landkarten hatten. }\end{array}$} \\
\hline \multirow[t]{2}{*}{65} & $\begin{array}{l}\text {... dice que el Dios ha empezado a } \\
\text { hablarle en chino. }\end{array}$ & $\begin{array}{l}\text {..., dass Gott in [sic] Chinesisch zu ihm } \\
\text { gesprochen hat... }\end{array}$ \\
\hline & \multicolumn{2}{|c|}{$\begin{array}{l}\text { Während im Spanischen unverständliche Sprache den Chinesen angelastet wird, ist } \\
\text { das im Deutschen weniger üblich und es hätte sich eine kreativere Übersetzungs- } \\
\text { lösung finden können. (Für Vorschläge zur Übersetzung von phraseologischen } \\
\text { Einheiten vgl. HENNEQUIN/GRUHN 2016.) }\end{array}$} \\
\hline 102 & $\begin{array}{l}\text { Los mayas tenían maíz hasta en la } \\
\text { sopa... }\end{array}$ & $\begin{array}{l}\text { Den Mais hatten die Mayas sogar in der } \\
\text { Suppe. Er wurde zum Frühstück... }\end{array}$ \\
\hline
\end{tabular}


Gruhn, D.H.; Cabrera, G.R.; Kottenhahn, K. - Touristen auf Yucatán

\begin{tabular}{|l|l|l|}
\hline & $\begin{array}{l}\text { Hasta en la sopa: einfach überall (ohne dass sich das konkret auf eine Suppe } \\
\text { beziehen muss). Da es in diesem Kapitel ums Essen geht, wird die Redewendung im } \\
\text { AT zu einem besonderen Sprachspiel. Im Deutschen entsteht der Eindruck, dass bei } \\
\text { den drei täglichen Mahlzeiten Maissuppe gegessen wurde. }\end{array}$ \\
\hline 102 & $\begin{array}{l}\text { Te estoy empezando a ver cara de } \\
\text { marzorca. }\end{array}$ & $\begin{array}{l}\text { Dein Gesicht sieht schon wie ein Maisfeld } \\
\text { aus. }\end{array}$ \\
\hline $\begin{array}{l}\text { Cara de mazorca = Maiskolbengesicht, in übertragener Bedeutung Pickelgesicht. Da } \\
\text { die Person den Kopf eines Maiskolbens hat, passt "Maisfeld" nicht mehr zum Bild. }\end{array}$ \\
\hline
\end{tabular}

In der englischsprachigen Version lassen sich ebenfalls denotative Übersetzungen von Redewendungen finden, wenn auch nicht so häufig wie in der deutschen:

\begin{tabular}{|l|l|l|}
\hline Seite & \multicolumn{1}{|c|}{} & \\
\hline 65 & $\begin{array}{l}\text {...dice que el Dios ha empezado a } \\
\text { hablarle en chino. }\end{array}$ & $\begin{array}{l}\text {...that God has begun to speak in } \\
\text { Chinese... }\end{array}$ \\
\hline & Eine englische Entsprechung wäre "it's all Greek to me". \\
\hline 102 & $\begin{array}{l}\text { Los mayas tenían maíz hasta en la } \\
\text { sopa... }\end{array}$ & The Maya even had maize soup. \\
\hline
\end{tabular}

Es gibt aber auch Beispiele sehr gelungener Übertragungen im Englischen, so auf Seite 34, wo ein Marktverkäufer einen Kunden ersticht und dazu ausruft: "Y es que cuando estoy en liquidación, ¡estoy en liquidación!” Der Witz liegt in der Doppeldeutigkeit von "liquidación”, das sowohl Ausverkauf als auch Liquidierung im Sinne von Beseitigung bedeuten kann. Im Englischen ist der Doppelsinn erhalten geblieben: "I'm going to make a killing with this sale" (to make a killing: ein Gemetzel veranstalten / einen Mordsgewinn machen).

\section{Zusammenfassende Bewertung der Übersetzungen und Ausblick}

Die Untersuchung der beiden Übersetzungen hat Folgendes ergeben: Der Verlag verfügte zum Zeitpunkt der Veröffentlichungen weder über die Software, noch über geschulte Lektoren, um fremdsprachige Texte zu verlegen. Beide Übersetzer scheinen keine Fachleute zu sein.

Die Übersetzung ins Deutsche:

- Weist zahlreiche inhaltliche, semantische, sprachliche, orthotypographische u.a. Verstöße auf. 


$$
\text { Gruhn, D.H.; Cabrera, G.R.; Kottenhahn, K. - Touristen auf Yucatán }
$$

- Stützt sich nicht auf Fachtexte oder sonstige deutschsprachige Paralleltexte.

- Zieht die Voraussetzungen der Zielgruppe nicht in Betracht und gibt dieser keinerlei unterstützende Informationen.

- Erfüllt ihre didaktische Funktion nur teilweise, da die zahlreichen Übersetzungs- und Layoutfehler Unglaubwürdigkeit bzw. Autoritätsverlust der Publikation erzeugen.

- Erfüllt ihre informative Funktion nur teilweise, da - neben dem Fehlen unterstützender Informationen - verschiedene Daten und Inhalte fehlerhaft übertragen wurden.

- Ein Übersetzungsprofil (im Sinne eines reflektierten Plans der Übersetzung) ist nicht zu erkennen.

Diese Übersetzung ist folglich nicht zielgruppenadäquat.

Die Übersetzung ins Englische:

- Weist nur geringfügige Abweichungen von der Normsprache (im Sinne von grammatisch und lexikalisch korrektem Sprachgebrauch der Zielsprache) auf; zeichnet sich aber durch einige Interferenzen aus dem Spanischen aus.

- Ist inhaltlich-semantisch weitgehend korrekt übertragen.

- Hat orthotypographische Schwächen mangels eines fachgerechten Layouts bzw. Lektorats.

- Zieht das Vorwissen der Zielgruppe ebenso wenig in Betracht wie die deutsche. Ein Übersetzungsprofil ist hier ebenfalls nicht zu erkennen.

In diesem Sinne kann auch die Übersetzung ins Englische nur mit Einschränkungen als zielgruppenadäquat bezeichnet werden.

Was den "Botschafter-Effekt" der zielsprachigen Veröffentlichungen betrifft, so kann vor allem die Übersetzung ins Deutsche einen Imageschaden bewirken. Das ist nicht so zu verstehen, dass die Leser nun von einer weiteren Mexikoreise absehen würden, es bedeutet vielmehr, dass dieser auf negative Weise zur Bildung eines Nationen-Images beitragen bzw. bereits bestehende Mexiko-Klischees bestätigt kann.

Die Verantwortung für die Veröffentlichung dieser Übersetzungen ist jedoch nur m. E. den "Übersetzern" anzulasten, die keine Fachleute sind und insofern ihre eigenen 


$$
\text { Gruhn, D.H.; Cabrera, G.R.; Kottenhahn, K. - Touristen auf Yucatán }
$$

übersetzerischen Fähigkeiten gar nicht sachgerecht einschätzen können. Die Hauptlast trägt der Verlag, der solche Übersetzungen (wohl unter dem Gesichtspunkt des geringen Kostenaufwands) in Auftrag gibt und verlegt - ohne sich mit übersetzerischen Kompetenzen und Studien zum Thema auseinandergesetzt zu haben. Wie Márquez (vgl. 2011: 53) ausführt, ist Zweisprachigkeit keinesfalls eine hinreichende Bedingung, die zur Übersetzung qualifiziert. Spezifische Kompetenzen müssen erlernt und erarbeitet werden. Ebenso könnte man von verlegerischen Kompetenzen im Bereich der Auftragsvergabe, der Qualitätskontrolle und dem Layout von Übersetzungen sprechen.

Während die Zahl der universitären Bachelor- und Masterstudiengänge im Bereich der Übersetzungswissenschaft wächst, sich die Fachkongresse mehren und translatorische Themen kontrovers diskutiert werden, ist es bei vielen Verlagen, aber auch Behörden oder Unternehmen immer noch gang und gäbe, Übersetzungsaufträge an Muttersprachler oder Absolventen eines Sprachkurses zu vergeben, die dafür gar nicht geschult sind.

So liegt auch im 21. Jahrhundert noch ein breiter Graben zwischen Übersetzungstheorie und berufsweltlicher Praxis. Die Fachwissenschaft, deren Gegenstand ja im Wesentlichen praxisbezogen ist, sollte sich diesem Problem stellen und versuchen, potentielle Auftraggeber von Übersetzungen stärker in ihre Diskussionen einzubeziehen.

\section{Literaturverzeichnis}

Covo TorRes, Javier. Los mayas en las rocas. Mérida, Yucatán: Editorial Dante, 1. Nachdruck, 2010.

Covo Torres, Javier. Die Mayas in der Steinzeit. Tradução de Kerstin Regel de Baena. Mérida, Yucatán, Editorial Dante, 2007.

COVO TORRES, Javier. The mayas on the rocks. Tradução de David Phillips. Mérida, Yucatán: Editorial Dante, 2010.

DURÁN MUÑOZ, Isabel. Caracterización de la traducción turística: problemas, dificultades y posibles soluciones. Revista de Lingüística y Lenguas Aplicadas, v. 7, p. 103-113, 2012. Disponível em: <https://dialnet.unirioja.es/ejemplar/372264>. Acesso em: 31 jul. 2017.

HeNNeQuin, Jean. En busca de la piedra traductorial. Tlaxcala/Puebla: Universidad Autónoma de Tlaxcala, El Colegio de Puebla, 1999.

HENNEQUin, Jean; GRUHN, Heike. Der Rückgriff auf Paralleltexte in der literarischen Übersetzung: Übersetzungskritik und Vorschläge bezüglich der Übersetzung von Sprichwörtern des Simplicissimus von Grimmelshausen. Verbum et Lingua, v. 7, p. 68-85, 2016. Disponível em: <http://verbumetlingua.cucsh.udg.mx/digital/7>. Acesso em 15 set. 2017.

KUPSCH-LOSEREIT, Sigrid. Interferenz in der Übersetzung. In: KITTEL, Harald et al. (Org.). Übersetzung. Translation. Traduction. Ein internationales Handbuch zur Übersetzungsforschung. 1. Teilband. Berlin; New York: Walter de Gruyter, 2004. p. 543-549. 
MARQUEZ, Daniel Alejandro. Las once competencias del traductor: El perfil ideal en servicios de traducción. Comunicación, Cultura y Política. Comunicación, cultura y política. Revista de Ciencias Sociales, n. 4, p. 53-68, 2011. Disponível em: <http://journal.ean.edu.co/index.php/revistai/article/view/659>. Acesso em: 10 ago. 2017.

NORD, Christiane. Textanalyse und Übersetzen. Heidelberg: Groos Verlag, 1991.

PARRA GALIANO, Silvia. Propuesta metodológica para la revisión de traducciones: principios generales y parámetros. TRANS, n. 11, p. 197-214, 2007. Disponível em: <http://www.trans.uma.es/trans_11.html>. Acesso em: 01 jul. 2017.

PREM, Hanns J. Die Azteken. Geschichte, Kultur, Religion. München: C.H.Beck, 2011.

RIESE, Berthold. Die Maya. Geschichte, Kultur, Religion. München: C.H.Beck, 2011.

SCHLEIERMACHER, Friedrich. Ueber die verschiedenen Methoden des Uebersezens. In: STÖRIG, Hans Joachim (Org.). Das Problem des Übersetzen, Stuttgart: H. Govers, 1963. p. 38-69.

SCHREIBER, Michael. Grundlagen der Übersetzungswissenschaft. Romanistische Arbeitshefte. Tübingen: Max Niemeyer Verlag, 2006.

VALERO GARCES, Carmen. La traducción del cómic: retos, estrategias y resultados. TRANS, n. 4, Universidad de Málaga, p. 75-88, 2000. Disponível em: <http://www.trans.uma.es/trans_04.html>. Acesso em: 1 out. 2017. 